\title{
CLASSICAL DIFFERENTIAL EQUATIONS ON MANIFOLDS
}

\author{
BY \\ L. AUSLANDER AND L. MARKUS
}

1. Types of differential objects on manifolds. Let $D$ be a differential object, say, the Laplace potential partial differential equation, the wave equation, the diffusion equation, or one of the corresponding differential operators defined in the Cartesian coordinates of Euclidean $n$-space $R^{n}$. We shall say that $D$ is well-defined on a differentiable manifold $M^{n}$ (connected, separable metric space with $C^{\infty}$ local coordinates) in case there is a differential equation or a differential operator defined on $M^{n}$, which in a certain atlas (covering of $M^{n}$ by a subcollection of the differentiable coordinate systems) is expressed by $D$.

The collection of all differentiable homeomorphisms of open sets of $R^{n}$ into $R^{n}$, which preserve $D$, is the pseudogroup of $D$, cf. [11]. Then we can state that $M^{n}$ admits the type of differential object $D$ if and only if $M^{n}$ has an atlas whose coordinate transition maps belong to the pseudogroup of $D$.

In this paper we shall find topological and geometrical properties of manifolds which admit the classical differential objects listed above. In the next section we relate this problem to the more standard one of reducing the structure group of the principal bundle of bases $B\left(M^{n}\right)$. In section three we study applications of the general theory to the Laplace and wave partial differential equations and also to linear ordinary differential equations with constant coefficients. Finally, in the appendix we state properties of bundle reductions to totally disconnected subgroups; these results being immediate generalizations of an earlier work of the authors [5] and of relevance for the present study.

2. Bundle reduction for the potential, wave, diffusion equations and operators. We note that the pseudogroup for the potential operator

$$
\nabla^{2} u=\partial^{2} u / \partial x_{1}^{2}+\cdots+\partial^{2} u / \partial x_{n}^{2},
$$$$
n \geqq 2 \text {, }
$$

is the Euclidean pseudogroup $\varepsilon_{n}$ consisting of the rigid motions (or their restrictions) of open sets in $R^{n}$, cf. [12, p. 18]. For the potential equation

$$
\nabla^{2} u=0
$$

the pseudogroup $\mathcal{P}_{n}$ is generated by the rigid motions and similarities, for $n \geqq 3$, but $P_{2}$ consists of holomorphic and conjugate holomorphic maps, cf. $[12$, p. 85$]$.

For the wave operator

Received by the editors October 1, 1956 and, in revised form, June 3, 1957. 


$$
\square^{2} u=\partial^{2} u / \partial x_{1}^{2}+\cdots+\partial^{2} u / \partial x_{n-1}^{2}-\partial^{2} u / \partial x_{n}^{2}, \quad n \geqq 2,
$$

one has the Lorentz pseudogroup $\mathfrak{L}_{n}$ consisting of Lorentz affine transformations of $R^{n},[12$, p. 18]. The wave equation

$$
\square^{2} u=0
$$

has the pseudogroup $\mathscr{W}_{n}$, which, for $n \geqq 3$, is generated by maps of $\mathscr{L}_{n}$ and similarities. For $n=2$ one can easily compute that the maps of $W_{2}$ are those for which the Jacobian matrices are everywhere in the group generated by the scalar matrices, the Lorentz matrices, and the transposition

$$
\left(\begin{array}{ll}
0 & 1 \\
1 & 0
\end{array}\right)
$$

The diffusion operator

$$
-\partial u / \partial x_{1}+\partial^{2} u / \partial x_{2}^{2}+\cdots+\partial^{2} u / \partial x_{n}^{2}, \quad n \geqq 2,
$$

has the pseudogroup $\varepsilon_{n, 1}$ which can easily be computed to consist of maps

$$
x^{1} \rightarrow x^{1}+\text { const., } \quad x^{\alpha} \rightarrow \underset{a_{\beta} x^{\beta}}{\alpha}+b^{\alpha}
$$

where $\left(a_{\beta}^{\alpha}\right) \in O(n-1)$ for constants $a_{\beta}^{\alpha}, b^{\alpha}$, where $\alpha, \beta=2,3, \cdots, n$. The pseudogroup $\mathscr{D}_{n}$ for the diffusion equation consists of the maps of $\varepsilon_{n, 1}$ and their compositions with the positive similarities.

The ordinary differential system

$$
\frac{d^{2} x^{i}}{d t^{2}}=0
$$$$
i=1,2, \cdots, n
$$

has the affine pseudogroup $Q_{n}$ which consits of the affinities of $R^{n}$.

We omit the direct calculations, most of which occur in the listed references, leading to the above descriptions of the various pseudogroups.

The principal theorem of this section relates the problem of constructing the potential, wave, diffusion equations and operators on manifolds $M^{n}$ to a reduction of the structure group $G L(n, R)$ of $B\left(M^{n}\right)$ to the scalar $C(n)$, orthogonal $O(n)$, and Lorentz $L(n)$ groups.

THEOREM 1. Let $M^{n}, n \geqq 2$, be a differentiable manifold. The existence of an atlas on $M^{n}$ compatible with the following pseudogroups is equivalent to a realizable reduction of the bundle of bases $B\left(M^{n}\right)$ to the indicated matrix group:

(a) $\mathfrak{Q}_{n}, n \geqq 2$, totally disconnected subgroup of $G L(n, R)$,

(b) $\mathcal{E}_{n}, n \geqq 2$, totally disconnected subgroup of $O(n)$,

(c) $\mathfrak{L}_{n}, n \geqq 2$, totally disconnected subgroup of $L(n)$,

(d) $\mathcal{P}_{n}, n \geqq 3$, totally disconnected subgroup of $C(n) \times O(n)$,

(e) $W_{n}, n \geqq 3$, totally disconnected subgroup of $C(n) \times L(n)$, 
(f) $\varepsilon_{n, 1}, n \geqq 2$, totally disconnected subgroup of $O(n-1)$,

(g) $D_{n}, n \geqq 2$, totally disconnected subgroup of $C(n) \times O(n-1)$,

(h) $\mathrm{P}_{2} \quad C(2) \times O(2)$,

(i) $\mathscr{W}_{2}$ group generated by

$$
C(2) \times L(2), \quad\left(\begin{array}{ll}
0 & 1 \\
1 & 0
\end{array}\right) .
$$

Proof. For an affine atlas the Jacobian transition matrices are constant on each connected intersection of local coordinate systems. Thus the structure group $G L(n, R)$ of $B\left(M^{n}\right)$ is reduced to a countable, and so, totally disconnected subgroup of $G L(n, R)$. Moreover this reduction is realized by the coordinate systems which form the affine atlas. Conversely, if a collection of local coordinate systems of $M^{n}$ realizes a reduction of $B\left(M^{n}\right)$ to a totally disconnected group, the Jacobian transition matrices are constant on each connected intersection of the coordinate systems. Thus the coordinate transition maps belong to the affine pseudogroup. So part (a) is proved.

All the remaining cases follow easily from the explicit descriptions of the pseudogroups at the beginning of this section.

In ( $\mathrm{f})$ and $(\mathrm{g})$ the notation means that we consider $O(n-1)$ as the orthogonal matrices in $O(n)$ for which the upper left corner element is +1 . Since $P_{2}$ and $\mathscr{W}_{2}$ are not subpseudogroups of $Q_{n}$, the corresponding bundle reductions need not lead to totally disconnected groups. Q.E.D.

The realizable bundle reductions indicated in Theorem 1 are easily recognized as classical structures of tensor calculus. For example, $M^{n}$ is an affine (a), Euclidean (b), or Lorentz (c) manifold if and only if $M^{n}$ admits respectively a flat (torsion and curvature zero) affine connection, a flat Riemannian metric tensor, a flat Lorentz tensor $\left(g_{i j}\right.$ with signature $+++\cdots+-$ ). Also the atlas $\varepsilon_{n, 1}$ can occur on $M^{n}$ if and only if $M^{n}$ is a Euclidean manifold with a constant nonzero vector field.

As examples we note that only the torus $T^{2}$, among all compact orientable 2-manifolds, admits any of the structures of Theorem 1 and furthermore that $T^{n}, n \geqq 2$, admits all those there mentioned. However, every $M^{2}$ admits the atlas of (h).

Among compact $n$-manifolds, $n \geqq 3$, no $M^{n}$ admits any of the atlases of Theorem 1 if the fundamental group $\pi_{1}\left(M^{n}\right)$ is finite, [5].

The conformal pseudogroup $\mathfrak{C}_{n}, n \geqq 2$, consisting of maps with Jacobians in $C(n) \times O(n)$, plays an important role in the study of classes of elliptic partial differential equations, as distinct from the single Laplace equation, on manifolds. If there is a differential equation on $M^{n}$ which has the form

$$
\nabla^{2} u+f(x, u, \partial u / \partial x)=0
$$

in each coordinate system of an atlas (here the local function $f$ depends on the coordinate system), then the atlas is compatible with $\mathfrak{e}_{n}$; that is, realizes 
a reduction of the group of $B\left(M^{n}\right)$ to $C(n) \times O(n)$. Next we note the converse of this proposition.

THEOREM 2. Let $M^{n}$ be a differentiable manifold with realizable reduction of $B\left(M^{n}\right)$ to $C(n) \times O(n), n \geqq 2$. Then there is an elliptic partial differential equation on $M^{n}$ which, in each coordinate system of the conformal atlas, has the form

$$
\nabla^{2} u+f_{(x)}(x, \partial u / \partial x)=0
$$

(where $f_{(x)} \in C^{\infty}$ depends on the local coordinate system).

Proof. Since $\partial u / \partial x^{i}=\left(\partial u / \partial y^{j}\right)\left(\partial y^{j} / \partial x^{i}\right)$, for a real differentiable function $u$ in overlapping coordinates where $y=y(x)$, we note that $f_{(x)}$ is actually a differentiable function defined on the cotangent vector bundle $T^{*}\left(M^{n}\right)$ over the coordinate patch $(x)$. We use the coordinates on $T^{*}\left(M^{n}\right)$ arising from the conformal atlas on $M^{n}$, say $(x)_{\alpha}$, and the differentials $d x_{\alpha}^{1}, \cdots, d x_{\alpha}^{n}$ to form an atlas on $T^{*}\left(M^{n}\right)$. Using this atlas we shall define a real line bundle $L$ over $T^{*}\left(M^{n}\right)$. The coordinate changes in $L$ are suggested by the transformations of $f_{(x)}$ under a conformal change of coordinates $x \rightarrow y(x)$ in $M^{n}$. Here

$$
f_{(y)}(y, \partial u / \partial y)=\frac{1}{K}\left[A^{j} \partial u / \partial y^{j}+f_{(x)}\left(x, \frac{\partial u}{\partial y^{j}} \frac{\partial y^{j}}{\partial x^{i}}\right)\right]
$$

where $K=\sum_{i=1}^{n}\left(\partial y^{j} / \partial x^{i}\right)^{2}, A^{j}=\sum_{i=1}^{n} \partial^{2} y^{i} / \partial x_{i}^{2}$.

Now let $(x, p)$ and $(y, q)$ be overlapping coordinate systems of $T^{*}\left(M^{n}\right)$, as described above. Let $z, w \in R$ and in the local product coordinates of $L$ form the identifications $(x, p, z)=(y, q, w)$ in case $y=y(x), p_{i}=q_{j} \partial y^{j} / \partial x^{i}$ and $w=K^{-1}\left[A^{i} q_{j}+z\right]$. Then a differentiable cross-section of $T^{*}\left(M^{n}\right)$ into the line bundle $L$ defines, for each conformal coordinate system $(x)$ of $M^{n}$, a function $f_{(x)}(x, \partial u / \partial x)$ compatible with the transformation formula for the coefficient of the partial differential equation.

Since the fiber $R$ is solid, the cross-section and thus the required elliptic partial differential equation exists on $M^{n}$. Q.E.D.

The next remark shows that for the study of a single differential equation the pseudogroup $\mathfrak{C}_{n}$ is too large.

REMARK. Let $\nabla^{2} u+f(x, u, \partial u / \partial x)=0$ with $f \in C^{\infty}$ for all real arguments, $x \in R^{n}$, have a pseudogroup $\mathcal{S} \supset \mathfrak{C}_{n}$. Then $n=2, \mathcal{G}=\mathfrak{C}_{2}$ and $f(x, u, \partial u / \partial x)$ $=g(u, \rho)$ where $\rho=|\nabla u|$ and $g \in C^{\infty}$ for $-\infty<u<\infty, \rho>0$. Also $\alpha^{-2} g(u, \alpha \rho)$ $=g(u, \rho)$ for each real $\alpha>0$. Conversely, every equation $\nabla^{2} u+g(u, \rho)=0$ with $g \in C^{\infty}$ on $-\infty<u<\infty, \rho>0$ and homogeneous of degree 2 in $\rho=|\nabla u|$, has pseudogroup $\mathcal{\Theta}=\mathrm{C}_{2}$.

The proof of these statements follows directly from the Liouville theorem that $\mathcal{C}_{n}$, for $n \geqq 3$, consists of rigid motions, similarities, and inversions in spheres. For hyperbolic partial differential equations

$$
\square^{2} u+f_{(x)}(x, u, \partial u / \partial x)=0
$$


the above Theorem 2 and the subsequent remark hold with minor modifications of replacing $C(n) \times O(n)$ by $C(n) \times L(n)$ and $\rho=|\nabla u|$ by

$$
\sigma=\left[\left(\partial u / \partial x_{1}\right)^{2}-\left(\partial u / \partial x_{2}\right)^{2}\right]^{1 / 2} .
$$

For the potential, wave, or diffusion equations we are led to a realizable reduction of $B\left(M^{n}\right)$ to a product group, with $C(n)$ as one factor. To define the corresponding differential operator on $M^{n}$ we need a further reduction which factors out the scalar matrices. To clarify this construction we shall define the unimodular obstruction.

Let $M^{n}$ be an affine manifold and for each closed continuous curve $\mathbb{5}$ on $M^{n}$ let $|\mathfrak{S}|$ be the absolute value of the determinant of the holonomy transformation around $\mathfrak{E}$. This is well-defined since the holonomy matrix for $\mathbb{E}$ is determined in $G L(n, R)$ up to similarity. Let $\gamma=\log |\mathfrak{\complement}|$ and extend $\gamma$ by linearity to the group of real, oriented, finite singular 1 -cycles. Since $\gamma$ is zero on bounding 1-cycles, $\gamma$ determines an element of the 1-dimensional, real, singular cohomology group of $M^{n}$.

Definition. Let $M^{n}$ be an affine manifold. The cohomology class $\gamma \in H^{1}\left(M^{n}, R\right)$ is called the unimodular obstruction of the affine atlas on $M^{n}$.

It is clear that on an affine manifold $M^{n}$ there exists a subordinate unimodular affine atlas if and only if $\gamma=0$. Also an affine manifold with atlas compatible with $\mathscr{P}_{n}, W_{n}$, or $\mathscr{D}_{n}$ has a subatlas compatible with $\mathcal{E}_{n}, \mathfrak{L}_{n}$, or $\mathcal{E}_{n, 1}$, respectively, if and only if $\gamma=0$. This occurs, of course, if $I I^{1}\left(M^{n}, R\right)=0$.

For if there is such a subordinate atlas then $\gamma=0$ since $O(n)$ and $L(n)$ are in the unimodular group. On the other hand, if $\gamma=0$ then, say for the case of a $P_{n}$ atlas, the holonomy group is in $O(n)$. Choose a local coordinate system $(x)$, centered at $P \in M^{n}$, of the $P_{n}$-atlas. For each point $Q \in M^{n}$ join $P$ to $Q$ by a differentiable curve $\mathcal{C}(t)$ and choose coordinate systems from the $\mathcal{P}_{n}$ atlas so that the carrier of $\mathfrak{C}(t)$ is covered and the Jacobian matrices of successive coordinate systems lie in $O(n)$. (We complete the affine $\boldsymbol{P}_{n}$-atlas if necessary.) In this manner construct a Euclidean subatlas on $M I^{n}$.

The next theorem shows that a manifold admitting the Laplace potential equation further admits, under a mild hypothesis, the Laplace operator.

THEOREM 3. Let $M^{n}$ be an affine manifold with atlas compatible with $\otimes_{n}$. If the flat affine connection $\Gamma$ of $M^{n}$ is complete, then $M^{n}$ has a subordinate Euclidean atlas.

Proof. Now $M^{n}$ can be considered as the affine space $R^{n}$ modulo a properly discontinuous group $\pi_{1}$ of affine transformations. Moreover, the homogeneous part of each map in $\pi_{1}$ is a matrix in $C(n) \times O(n)$. Suppose for some map $h \in \pi_{1}$ the scalar $C(n) \neq I$. Then $h\left(\right.$ or $h^{-1}$ ) must be a strict contraction of $R^{n}$, referred to the usual metric. But then $h$ has a fixed point in $R^{n}$ which is impossible. Thus $C(n)=I$ for each $h \in \pi_{1}$ and the holonomy group of $M I^{n}$ lies in $O(n)$. Therefore $M^{n}$ is a Euclidean manifold. Q.E.D. 
Corollary. Let $M^{n}$ be a compact affine manifold with $\rho_{n}$-atlas. Then $\gamma=0$ if and only if the flat affine connection $\Gamma$ on $M^{n}$ is complete.

Proof. If $\Gamma$ is complete on $M^{n}$, then $M^{n}$ is a Euclidean manifold and so $\boldsymbol{\gamma}=0$. Conversely, if $\boldsymbol{\gamma}=0$ then $M^{n}$ is a compact Euclidean manifold and so $\Gamma$ is complete. Q.E.D.

3. Harmonics on Euclidean manifolds and other applications. On a Euclidean manifold $M^{n}$ we can consider the Laplace-Beltrami differential operator, which, in the Euclidean atlas, has the form

$$
\nabla^{2} u=\partial^{2} u / \partial x_{1}^{2}+\cdots+\partial^{2} u / \partial x_{n}^{2} .
$$

We shall be interested in the harmonics or eigenfunctions for this operator, that is, real differentiable functions $u$ on $M^{n}$ for which

$$
\nabla^{2} u=\lambda u
$$

for some real constant $\lambda$ (we allow $\lambda=0$ ).

We restrict our attention to the compact Euclidean manifolds $M^{n}$, which are known to be finitely covered by the flat torus $T^{n}$.

Lemma 1. Let $M^{n}$ be a compact Euclidean manifold. Then the set of all finite linear combinations, with real coefficients, of eigenfunctions of the Laplace operator forms a real algebra $A_{M}$.

Proof. Let $M^{n}$ be covered by the flat torus $T^{n}$. Each eigenfunction of the Laplace operator on $M^{n}$ lifts to an eigenfunction of the Laplace operator on $T^{n}$. Now the eigenfunctions on $T^{n}$ are of the form $Q\left(x_{1}, \cdots, x_{n}\right)=\phi_{1}\left(x_{1}\right) \phi_{2}\left(x_{2}\right)$ $\cdots \phi_{n}\left(x_{n}\right)$ where

$$
\phi_{i}\left(x_{i}\right)=\sin \left(2 \pi m / l_{i}\right) x_{i} \text { or } \phi_{i}\left(x_{i}\right)=\cos \left(2 \pi m / l_{i}\right) x_{i}, \quad m=0,1,2, \cdots
$$

and $l_{i}$ is the length of the corresponding edge of the torus $T^{n}$. By elementary trigonometric identities the finite linear combinations of the eigenfunctions on $T^{n}$ form a real algebra $A_{T}$.

The covering transformation group of $T^{n}$ over $M^{n}$ is isomorphic with the holonomy group $H\left(\Gamma, M^{n}\right)$, and we fix this isomorphism. Since the map $P \rightarrow h(P)$ of $T^{n} \rightarrow T^{n}$, for $h \in H\left(\Gamma, M^{n}\right)$, is an isometry, $Q(h(P))$ is an eigenfunction for the Laplace operator on $T^{n}$ and for the same eigenvalue as is $Q(P)$. Thus the average $\sum_{h \in H\left(\Gamma, M^{n}\right)} Q(h(P))=Q^{*}(P)$ is an eigenfunction on $T^{n}$. Also $Q^{*}(x)$ projects to an eigenfunction of the Laplace operator on $M^{n}$.

Then each element of $A_{T}$ which is invariant under the action of $H\left(\Gamma, M^{n}\right)$ on $T^{n}$ can be written as a finite linear combination of eigenfunctions on $T^{n}$ each of which projects to an eigenfunction on $M^{n}$. Thus the subalgebra of $A_{T}$, consisting of those functions invariant under $H\left(\Gamma, M^{n}\right)$, projects onto the set $A_{M}$ of real linear combinations of eigenfunctions of $M^{n}$. Moreover this projection establishes an isomorphism of $A_{M}$, as a linear space of real func- 
tions on $M^{n}$, with a subalgebra of $A_{T}$. Moreover the isomorphism preserves products and so $A_{M}$ is a real algebra. Q.E.D.

The algebra $A_{M}$, considered as a commutative ring with a unit, has no zero divisors and so one can form the field of quotients $F_{M}$, called the eigenfunction field for the compact Euclidean manifold $M^{n}$. By lifting the functions on $M^{n}$ to the covering torus $T^{n}$, we shall of ten write $A_{M} \subset A_{T}$ and $F_{M} \subset F_{T}$.

LEMMA 2. The eigcnfunction ficld $F_{M}$, for a compact Euclidean manifold $M^{n}$, has transcendence degree $\left\{F_{M}: R\right\}=n$ over the real numbers.

Proof. By the general theory of harmonic analysis, one knows that each real differentiable function $f$ on $M^{n}$ can be approximated uniformly by elements of $A_{M}$. Choose a set $\psi_{1}(x), \cdots, \psi_{n}(x)$ of elements of $A_{M}$ which approximate, respectively, the local coordinate functions near $P \in M^{n}$. Also require that the gradients $\nabla \psi_{1}, \cdots, \nabla \psi_{n}$ form a basis for the tangent spaces to $M^{n}$ everywhere near $P$.

If the transcendence degree $\left\{F_{M}: R\right\}<n$ then there is a nontrivial polynomial relation

$$
p\left(\psi_{1}(x), \cdots, \psi_{n}(x)\right)=0 \text { on } M^{n} .
$$

But then one easily calculates that the gradients $\nabla \psi_{1}, \cdots, \nabla \psi_{n}$ are linearly dependent on some open set near $P$. This contradiction shows that

$$
\left\{F_{M}: R\right\} \geqq n \text {. }
$$

However, one can list explicitly a transcendence basis, say $\sin \left(2 \pi / l_{i}\right) x_{i}$ $(i=1,2, \cdots, n)$, for $F_{T}$ over the reals. Thus $\left\{F_{T}: R\right\}=n$ and since one can consider $F_{M} \subset F_{T}$, we have $\left\{F_{M}: R\right\} \leqq n$. Q.E.D.

Theorem 4. Let $M^{n}$ be a compact Euclidean manifold and let $F_{M}$ and $F_{T}$ be the eigenfunction fields for the Laplace operator on $M^{n}$ and on the covering torus $T^{n}$, respectively. Then $F_{T}$ is a finite, normal, algebraic extension of $F_{M} \subset F_{T}$ and the Galois group of $F_{T}$ over $F_{M}$ is isomorphic with the holonomy group $H\left(\Gamma, M^{n}\right)$.

Proof. Since $F_{M}$ and $F_{T}$ each have transcendence degree $n$ over the reals, $F_{T}$ is an algebraic extension of $F_{M}$. Moreover, $F_{T}$ is finitely generated over $R$ and thus over $F_{M}$ so that $F_{T}$ is a finite algebraic extension of $F_{M}$.

Consider the group (i) of all automorphisms of $F_{T}$ which leave $F_{M}$ elementwise fixed. For each element $h$ of the holonomy group $I I\left(\Gamma, M I^{n}\right)$ there is an isometry of $T^{n}$ onto itself. Since an isometry of $T^{n}$ preserves the Laplace operator, there is defined thereby an automorphism $g_{h}$ of $F_{T}$ onto itself. Thus there is a homomorphism $\Phi: h \rightarrow g_{h}$ of $I I\left(\Gamma, M^{n}\right)$ onto (i) ${ }_{I} \subset$ (3). We now show that $\Phi$ is an isomorphism. If $h$ is not the identity of $I I\left(\Gamma, M^{n}\right)$, then take a real differentiable function $f$ on $T^{n}$ separating the points $P$ and $h(P)$, for some $P \in T^{n}$. But $f$ is uniformly approximated by a function in $F_{T}$ which 
is thereby moved under $g_{h}$. Thus $g_{h}$ is the identity of $(s)$ if and only if $h$ is the identity of $H\left(\Gamma, M^{n}\right)$ and $\Phi$ is an isomorphism.

We next show that $F_{T}$ is a normal extension of $F_{M}$. Suppose a function $\psi \in F_{T}$ is invariant under the automorphisms of $(\$)$. Then $\psi$ is invariant under $\left(B_{H}\right.$. Now there exist functions $\rho(P), \sigma(P)$ in $A_{T}$ with $\rho(P) \psi(P)=\sigma(P)$. As in Lemma 1 average the terms of this equation for arguments $h(P), h \in H\left(\Gamma, M^{n}\right)$, to obtain $\rho^{*}(P) \psi(P)=\sigma^{*}(P)$. Here $\rho^{*}(P)$, and $\sigma^{*}(P)$ lie in $A_{T}$ and are invariant under $\mathfrak{\Im j}_{H}$ and so, by Lemma $1, \rho^{*}, \sigma^{*} \in A_{M}$. Thus $\psi \in F_{M}$ and $F_{T}$ is a normal extension of $F_{M}$.

Let $F_{H}$ be the subfield of $F_{T}$ which corresponds to the subgroup $\mathfrak{B S}_{H}$. Then $F_{T}$ is normal over $F_{H}$ with Galois group $B_{H}$. But, as shown above, $F_{H}=F_{M}$. Thus $B=B_{H}$. Q.E.D.

It is of interest to examine the dimensions of the eigenspaces for the Laplace operator on a compact Euclidean manifold $M^{n}$. For convenience we modify $M^{n}$, within similarity, so that the covering flat torus has edges of length $2 \pi$.

THEOREM 5. For the flat torus $T^{n}$, with edges of length $2 \pi$, the eigenvalues for the Laplace operator are negative integers (and zero) and this is also the case for each compact Euclidean manifold $M^{n}$ covered by $T^{n}$. If $n>4$ every negative integer occurs as an eigenvalue on $T^{n}$.

Proof. For a compact Euclidean manifold $M^{n}$ the eigenvalues of the Laplace operator are among those of the covering torus. The eigenfunctions for this torus, with edges of length $2 \pi$, are $\phi_{1}\left(x_{1}\right) \phi_{2}\left(x_{2}\right) \cdots \phi_{n}\left(x_{n}\right)$ where $\phi_{i}\left(x_{i}\right)=\sin m x_{i}$ or $\cos m x_{i}(m=0,1,2, \cdots)$. Thus the eigenvalues for the Laplace operator on $T^{n}$ are $-\left(m_{1}^{2}+m_{2}^{2}+\cdots+m_{n}^{2}\right)$. If $n \geqq 4$, one knows that every positive integer can be represented as the sum of four squares and so every negative integer is an eigenvalue on $T^{n}$. Q.E.D.

It is easy to answer some arithmetic problems concerning the eigenvalues and the eigenspaces by standard number theoretic arguments. The next remark illustrates this technique.

COROLlARy. If $n=4$, the multiplicity of the eigenvalue $-m$, for the Laplace operator on the flat torus $T^{n}$ with edges of length $2 \pi$, is $8 \Sigma$ where $\Sigma$ is the sum of all divisors of $m$ which are not multiples of 4 .

Proof. We consider the number of representations of a positive integer $m$ as the sum of four squares. Allowing for the two eigenfunctions $\sin m_{i} x_{i}$ and $\cos m_{i} x_{i}$ with eigenvalue $-m_{i}^{2}$, one obtains the required conclusion as a result in the number theory [13]. Q.E.D.

The harmonics of the Laplace equation arise classically in the solution of the wave partial differential equation. The wave equation is well-defined on a Lorentz manifold, that is, a differentiable manifold $M^{n}$ with a $\mathfrak{L}_{n}$-atlas. 
If initial data for the Cauchy problem for the wave equation are prescribed on a space-like hypersurface in $M^{n}$, then a differentiable solution function can be constructed in some neighborhood of the initial surface. However, it is not always possible to extend this solution throughout $M^{n}$. The next theorem analyzes this problem in a special simple case.

Consider the product $M^{n}$ of a Euclidean manifold $M^{n-1}$ and a metric circle $S^{1}$. Then there is a natural flat Lorentz tensor on $M^{n}$ defined by the product metric except that the vectors tangent to $S^{1}$ are taken with a negative "norm."

THEOREM 6. Consider the product manifold $M^{n}$ of a compact, orientable, Euclidean manifold $M^{n-1}$ and a metric circle $S^{1}$. Let $M^{n}$ have the natural Lorentz atlas. Then $M^{n}$ admits no nonconstant differentiable solution for the wave equation unless the radius of $S^{1}$ lies in a certain countable set which depends on $M^{n-1}$.

Proof. Each solution of the wave equation on $M^{n}$ lifts to a solution on $M^{n-1} \times R^{1}$ which has period $d$ in time, where $d$ is the circumference of $S^{1}$.

The only solutions of the wave equation on $M^{n}$, which are a product of functions on $M^{n-1}$ and on $S^{1}$ are

$$
\begin{aligned}
& \phi_{m}(P) \sin \left(-\lambda_{m}\right)^{1 / 2} t, \\
& \phi_{m}(P) \cos \left(-\lambda_{m}\right)^{1 / 2} t
\end{aligned}
$$

where $\phi_{m}(P)$ are eigenfunctions for the Laplace operator on the Euclidean manifold $M^{n-1}$ and which correspond to the eigenvalues $\lambda_{m}, m=1,2, \cdots$. The $-\lambda_{m}$ are non-negative integers and each eigenspace has finite dimension. We take the set $\phi_{m}(P)$ to be orthonormal and complete in the real Hilbert space $L_{2}\left(M^{n-1}\right)$ and thus some of the values of $\lambda_{m}$ are repeated a finite number of times.

A differentiable solution $\psi(P, t)$ of the wave equation on $R^{1} \times M^{n-1}$ must have a uniformly convergent series expansion

$$
\psi(x, t)=\sum_{m=1}^{\infty} \phi_{m}(P)\left[A_{m} \cos \left(-\lambda_{m}\right)^{1 / 2} t+B_{m} \sin \left(-\lambda_{m}\right)^{1 / 2} t\right] .
$$

Then for each fixed $P \in M^{n-1}, \psi$ is an almost periodic function of $t$ with a modulus generated (over the integers) by $\left(-\lambda_{m}\right)^{1 / 2}, m=1,2, \ldots$. But $\psi$ has period $d$ in time and this is possible only if $2 \pi / d$ lies in the modulus generated by the countable set of real numbers $\left(-\lambda_{m}\right)^{1 / 2}, m=1,2, \cdots$ Q.E.D.

As our last example of the general theory of the preceding section, we consider some examples of linear differential equations with constant coefficients on affine manifolds.

Consider an affine vector field $V$ or linear differential equation with constant coefficients 


$$
V: \dot{x}^{i}=a_{j}^{i} x^{j}+b^{i}, \quad i=1,2, \cdots, n
$$

where $\left(a_{j}^{i}\right)$ and $\left(b^{i}\right)$ are real numbers depending on the affine coordinate system on the affine manifold $M^{n}$.

THEOREM 7. Let $V$ be a linear differential equation with constant coefficients on a compact affine manifold $M^{n}$ having a complete flat affine connection. Then the manifold of critical points $Z$ is either empty or $Z=M^{n}$. In the first instance $V$ is a constant (nonzero) vector field and in the second instance $V$ is the zero vector field.

Proof. Suppose $Z$ is nonempty. Then the lifted manifold $\tilde{Z} \subset \tilde{M}^{n}=R^{n}$ is an affine subspace of dimension $m, 0<m \leqq n$. If $m<n$, then each element of $\pi_{1}$, considered as a properly discontinuous group of affine transformations of $R^{n}$, has a translation vector parallel to $\tilde{Z}$. But then one could select an infinite set of points $Q_{i}$, each of which lies in a different hyperplane $\tilde{Z}_{i}$ parallel to $\tilde{Z}$ in $R^{n}$. But then there is no point in $R^{n}$ which is an accumulation point for the points of the orbits of the $Q_{i}$ and this contradicts the fact that $M^{n}$ is compact. Therefore $m=n$ and $V$ is the zero vector field on $M^{n}$.

In the first instance where $Z$ is empty, $\tilde{Z}$ is empty. We show that the lifted vector field $\tilde{V}$, above $V$, is constant in $R^{n}$. Consider the rank $r$ of the homogeneous terms in the vector field $\tilde{V}$. There is a point in $R^{n}$ where the rank is a minimum and the set of points in $R^{n}$ where this minimal rank is attained is an affine subspace $\tilde{Z}_{r}$ in $R^{n}$. Since $\tilde{Z}_{r}$ is invariant under $\pi_{1}$, we follow the previous argument concerning $\tilde{Z}$, and conclude that $\tilde{Z}_{r}=R^{n}$. Therefore the rank of $\tilde{V}$ is a constant. Thus $\tilde{V}$ is a constant vector field on $R^{n}$ and $V$ is constant on $M^{n}$. Q.E.D.

Thus to construct nontrivial linear differential equations with constant coefficients we must consider incomplete affine-manifolds. The following examples show that such differential equations can have new interesting types of solutions, for instance, isolated periodic solutions.

EXAmple. Consider the affine quarter-plane $x>0, y>0$ under the properly discontinuous transformation group generated by the powers of $T_{1}$ : $(x, y) \rightarrow(2 x, y)$ and $T_{2}:(x, y) \rightarrow(x, 2 y)$. The quotient space is an affine torus which admits no constant vector fields; although it does admit two linearly independent vector fields with constant cocfficients.

One can remark that if a compact affine manifold $M^{n}$ admits a subordinate unimodular atlas then each constant vector field $V$ on $M^{n}$ defines a measurepreserving flow. Since the measure of $M^{n}$ is then finite, almost all points $P \in M^{n}$ are $( \pm)$-stable à la Poisson.

EXAmple. We conclude this section with a significant example in which we shall describe the qualitative form of every noncritical differential equation with constant coefficients on the affine torus $M^{2}$ constructed from the punctured plane under the iterates of $T:(x, y) \rightarrow(2 x, 2 y)$. Such a linear differ- 
ential equation $V$ on $M^{2}$ lifts to a system

$$
\begin{aligned}
\tilde{V}: \dot{x} & =a x+b y+c, \\
\dot{y} & =e x+f y+h
\end{aligned}
$$

on the punctured plane. Since $\tilde{V}$ is invariant under $T, c=h=0$. Now perform a linear change in variables in the punctured plane, which of course commutes with the scalar matrix of $T$, so as to reduce $\widetilde{V}$ to a simplified form. In effect, we can require that the coefficient matrix of $\tilde{V}$ shall be in real Jordan canonical form. There are five cases to be discussed.

CASE 1 . $\dot{x}=\lambda_{1} x, \dot{y}=\lambda_{2} y$ where $\lambda_{1} \lambda_{2}<0$. This yields a saddle point in $R^{2}$. On $M^{2}$ there are four periodic solution curves and every other solution spirals towards some one of these with each of its ends.

CAse 2a. $\dot{x}=\lambda_{1} x, \dot{y}=\lambda_{2} y$ with $\lambda_{1} \lambda_{2}>0, \lambda_{1} \neq \lambda_{2}$. This is a node in $R^{2}$ but the qualitative behavior of the solutions on $M^{2}$ is the same as in Case 1.

CASE 2 b. $\dot{x}=\lambda x, \dot{y}=\lambda y$. This is a radial node in $R^{2}$. On $M^{2}$ each solution curve is periodic.

CASE 3. $\dot{x}=\lambda x+y, \dot{y}=\lambda y$. This is a one-sided node in $R^{2}$. On $M^{2}$ there are two periodic solutions and every other solution curve spirals towards one of these with each of its ends.

CASE 4 . $\dot{x}=y, \dot{y}=-(a f-b e) x+(a+f) y$ where $(a+f)^{2}-4(a f-b e)<0$. This is a spiral point in $R^{2}$. On $M^{2}$ one has two possible alternatives; either every solution curve is dense in $M^{2}$, or else every solution curve is periodic. The latter case occurs when the pitch of the spiral in $R^{2}$ is a multiple of 2 , that is, if and only if

$$
\frac{2(a+f)}{\left[4(a f-b e)-(a+f)^{2}\right]^{1 / 2}}=n \log 2 \text { for some } n=0, \pm 1, \pm 2, \cdots .
$$

Case 5. $\dot{x}=y, \dot{y}=-(a f-b c) x$ where $-(a f-b e)<0$. This is a center in $R^{2}$ and in $M^{2}$ each solution curve is periodic.

Finally we consider the paths, nonconstant solutions of $\ddot{x}^{i}=0, i=1,2$, $\cdots, n$, on $M^{n}$, an affine manifold with flat affine connection $\Gamma$. It is an clementary, but important, remark that on an affine manifold $M^{n}$, with a complete flat affine connection $\Gamma$, each pair of points can be joined by a path. This property of complete flat affine connections holds always for affine connections arising from a complete Riemannian metric but is not generally valid for a complete, symmetric affine connection on a compact differentiable manifold.

On a compact Riemannian manifold there is a periodic geodesic in each free homotopy class. The next example describes an affine torus $T^{n}$, with complete flat affine connection $\Gamma$, and which has no periodic paths. Consider the following $n$ affine maps of $R^{n}$, cf. [14]. 


$$
\begin{aligned}
& T_{1}: y_{1}=x_{1}+q x_{2}+q x_{3}+\cdots+q x_{n}+\lambda \text {, } \\
& y_{2}=x_{2}+q, \\
& \begin{array}{lll}
y_{3}= & x_{3} & +q,
\end{array} \\
& \text {. } \\
& \text {. } \\
& y_{n}=\quad x_{n}+q, \\
& T_{\alpha}: y_{1}=x_{1}+x_{\alpha} \text {, } \\
& y_{i}=x_{i} \quad i \equiv \alpha, \\
& y_{\alpha}=x_{\alpha}+1 \text {, } \\
& \alpha=2,3, \cdots, n \text {. }
\end{aligned}
$$

Here $q>0$ is irrational and $\lambda>q^{2}$.

One computes that these $n$ affinities generate a free abelian group with the general transformation given by:

$$
\begin{aligned}
& y_{1}=x_{1}+\sum_{\alpha=2}^{n}\left(m_{\alpha}+s q\right) x_{\alpha}+\lambda_{G}, \\
& y_{\alpha}=x_{\alpha}+\left(m_{\alpha}+s q\right)
\end{aligned}
$$

for integers $m_{\alpha}$ and $s$, and

$$
\lambda_{G}=\sum_{\alpha=2}^{n} \frac{1}{2}\left(m_{\alpha}+s q\right)^{2}+s\left(\lambda-q^{2}\right)-\frac{1}{2} \sum_{\alpha=2}^{n} m_{\alpha} .
$$

Moreover this group of affinities acts as a properly discontinuous group on $R^{n}$ and the quotient space is an affine manifold $M^{n}$ with a complete flat affine connection.

We show next that $M^{n}$ is compact by demonstrating that the fundamental domain is bounded. Given a point $\left(x_{1}, \cdots, x_{n}\right)$ in $R^{n}$ one can apply the maps $T_{\alpha}$ so as to consider only the case where $\left|x_{\alpha}\right| \leqq 1, \alpha=2, \cdots, n$. Then solve the equations (over the reals)

$$
\begin{aligned}
& 0=x_{1}+\sum_{\alpha=2}^{n}\left(m_{\alpha}+s q\right) x_{\alpha}+\lambda_{G}, \\
& 0=x_{\alpha}+\left(m_{\alpha}+s q\right)
\end{aligned}
$$

to obtain the real numbers:

$$
\begin{array}{rlrl}
s & =\left[\frac{1}{2} \sum_{\alpha=2}^{n}\left(x_{\alpha}^{2}-x_{\alpha}\right)-x_{1}\right]\left[\left(\lambda-q^{2}\right)+\frac{n-1}{2} q\right]^{-1}, \\
m_{\alpha} & =-\left(x_{\alpha}+s q\right), & \alpha=2,3, \cdots, n .
\end{array}
$$

Now modify these values of $s$ and $m_{\alpha}$ to the nearest integer values and then the resulting values of $\left(y_{1}, \cdots, y_{n}\right)$ will lie within a fixed (independent of the 
$\left.\left(x_{1}, \cdots, x_{n}\right)\right)$ bounded neighborhood of the origin.

It is easy to check that, for the general transformation of the group, the translation vector is not an eigenvector of the homogeneous part. Thus there are no closed periodic paths on $M^{n}$. A more careful analysis shows that $M^{n}$ is topologically the torus $T^{n}$.

4. Appendix on bundle reductions to totally disconnected groups. In a previous work [5] the authors investigated realizable reductions of $B\left(M^{n}\right)$ to totally disconnected subgroups of $G L(n, R)$. Here we recall this theory but in a quite general setting. The proofs of the following statements will be omitted since they parallel closely those in [5].

Consider a principal fiber bundle $P\left(M^{n}\right)$ over $M^{n}$ with Lie group $G$. Then $P\left(M^{n}\right)$ is reduced to a subgroup $K \subset G$ in case a left coset $K_{P}$ is distinguished in the fiber above each point $P \in M^{n}$ and, using the local product structure over a local coordinate system $V_{\alpha}$, there is a differentiable map $V_{\alpha} \rightarrow G: Q \rightarrow k_{Q}\left(V_{\alpha}\right) \in K_{Q}$. This is the usual definition [17] except that we do not require $K$ to be closed in $G$. If $P\left(M^{n}\right)=B\left(M^{n}\right)$ an equivalent definition was given by Chern [10] in terms of differential forms. Here one designates in each local coordinate system $V_{\alpha}$ a basis for the covector spaces, $\theta_{\alpha}^{1}, \theta_{\alpha}^{2}, \cdots$, $\theta_{\alpha}^{n}$. On each nonempty intersection $V_{\alpha} \cap V_{\beta}$ one has $\theta_{\alpha}^{i}=k_{\alpha \beta, j}^{i}(Q) \theta_{\beta}^{j}$ for $Q$ $\in V_{\alpha} \cap V_{\beta}$ and the matrices $k_{\alpha \beta, j}^{i}(Q)=k_{\alpha \beta}(Q) \in K$. If, furthermore, $\theta_{\alpha}^{i}=d x_{\alpha}^{i}$ in the coordinates $V_{\alpha}=(x)_{\alpha}$, then the reduction is realized.

In the general case of a principal bundle $P\left(M^{n}\right)$ the defining fields $k_{Q}\left(V_{\alpha}\right)$ yield distinguished coordinates in $P\left(M^{n}\right)$. The "generalized Jacobians" $J_{\alpha \beta}(Q) \in K$ are then defined by $J_{\alpha \beta}(Q) \eta_{\alpha}=\eta_{\beta}$ where $\left(\eta_{\alpha}, x_{\alpha}\right)$ and $\left(\eta_{\beta}, x_{\beta}\right)$ are the coordinates of the same element in $P\left(M^{n}\right)$.

Let a principal fiber bundle $P\left(M^{n}\right)$ over a differentiable manifold $M^{n}$, with Lie group $G$, be reduced to a subgroup $K \subset G$. If $K$ is totally disconnected, then the generalized Jacobians $J_{\alpha \beta}(Q)$ are constant on each component $Q \in V_{\alpha} \cap V_{\beta}$. Conversely, if the $J_{\alpha \beta}$ are constants on each component of $V_{\alpha} \cap V_{\beta}$, then $P\left(M^{n}\right)$ can be reduced to a totally disconnected subgroup $K^{\prime} \subset K$.

Let $P\left(M^{n}\right)$ be reduced to a totally disconnected group $K$ as above. Now re-topologize $K$ to be discrete and then the collection of left cosets $K_{P}$, for $P \in M^{n}$, form a covering space $K\left(M^{n}\right)$ over $M^{n}$. Actually $K\left(M^{n}\right)$ is a principal fiber bundle over $M^{n}$ with discrete group $K$ and projection $\pi: K\left(M^{n}\right) \rightarrow M^{n}$. Each component of $K\left(M^{n}\right)$ is a differentiable manifold so that $\pi$ is a differentiable map.

We define a connection $\Gamma$ for $M^{n}$ by prescribing every tangent vector in $K\left(M^{n}\right)$ as horizontal [1]. Then $\Gamma$ is flat, that is, has zero curvature but may have torsion if $P\left(M^{n}\right) \neq B\left(M^{n}\right)$. Using the appropriate concept of parallel displacement of the fiber along piecewise differentiable curves in $M^{n}$, one defines two such curves in $M^{n}$ to be holonomous in case they join the same initial and final points and yield the same parallel displacement of the fiber. Actually, the introduction of $\Gamma$ is rather artificial and parallel displacement 
can be defined directly in terms of the generalized Jacobians of the bundle reduction. Let $\subseteq(t), t_{1} \leqq t \leqq t_{2}$, be a continuous curve in $M^{n}$ joining points $P$ and $Q$. Choose a finite collection of local coordinate systems $P \in V_{\alpha}, V_{\alpha_{1}}$, $V_{\alpha_{2}}, \cdots, V_{\alpha_{m}}, Q \in V_{\beta}$ which overlap consecutively and cover the carrier of $\mathfrak{C}(t)$. Then if $\eta$, above $P$, has coordinate $\eta_{\alpha}$, that is, $\eta=k_{P}\left(V_{\alpha}\right) \eta_{\alpha}$, then in the coordinates of $V_{\beta}$ the parallel displacement of $\eta$ along $\Subset(t)$ is $k_{Q}\left(V_{\beta}\right) \eta_{\beta}$. Here the formula for parallel displacement is $\eta_{\beta}=J_{\alpha_{m} \beta} \cdots J_{\alpha_{1} \alpha_{2}} J_{\alpha \alpha_{1}} \eta_{\alpha}$.

The holonomy group $H\left(M^{n}, \Gamma\right)$ is the set of holonomy equivalence classes of closed, piecewise differentiable, curves, based at some point $P \in M^{n}$, with the usual groupoid multiplication. It is clear that holonomy groups of $M^{n}$ based at different points are isomorphic, in fact, by an isomorphism determined up to an inner automorphism.

Let $\pi_{1}\left(M^{n}\right)$ be the fundamental group of a differentiable manifold $M^{n}$. Let $\Gamma$ be the flat connection for a principal fiber bundle $K\left(M^{n}\right)$ with a discrete group $K$. Let $H\left(M^{n}, \Gamma\right)$ be the holonomy group based at the same point as is $\pi_{1}\left(M^{n}\right)$. Then there is a homomorphism of $\pi_{1}\left(M^{n}\right)$ onto $H\left(M^{n}, \Gamma\right)$.

Of course, if $\pi_{1}\left(M^{n}\right)=0$, then $K\left(M^{n}\right)$ is equivalent to a product bundle and each component of $K\left(M^{n}\right)$ is homeomorphic with $M^{n}$. In this case $H\left(M^{n}, \Gamma\right)=0$.

Definition. Let a principal fiber bundle $P\left(M^{n}\right)$ over a differentiable manifold $M^{n}$ be reduced to a totally disconnected group $K$. A component of the corresponding covering space $K\left(M^{n}\right)$ is called the holonomy covering space $\hat{M}^{n}$ of $M^{n}$.

The holonomy covering space $\hat{M}^{n}$ is unique in the sense that any two coverings of $M^{n}$ by components of $K\left(M^{n}\right)$ are equivalent. Furthermore, $\hat{M}^{n}$ could also be defined as the holonomy equivalence classes of piecewise differentiable curves in $M^{n}$, initiating at a point $P \in M^{n}$ and topologized as is customary in the theory of covering spaces. It is evident that, with the raised connection $\widehat{\Gamma}$, the holonomy group $H\left(\hat{M}^{n}, \widehat{\Gamma}\right)=0$.

Let $\hat{M}^{n}$ be the holonomy covering space of the differential manifold $M^{n}$, arising from a reduction of $P\left(M^{n}\right)$ to a totally disconnected group $K$. Let $\left(M^{0}, \Pi^{0}\right)$ be a covering space of $M^{n}$ and let $\Gamma^{0}$ be the connection on $M^{0}$ above the flat connection $\Gamma$ on $M^{n}$. Then $\left(M^{0}, \Pi^{0}\right)$ covers $\left(\hat{M}^{n}, \widehat{\mathrm{II}}\right)$ over $M^{n}$ if and only if $H\left(M^{0}, \Gamma^{0}\right)$ $=0$.

If $B\left(M^{n}\right)$ is reduced to a totally disconnected subgroup $K$, then one can define parallel displacements of the tangent spaces of $M^{n}$, along piecewise differentiable curves in $M^{n}$, by means of the generalized Jacobians described above. Also, for the flat connection $\Gamma$, one can define a path as a differentiable curve whose tangent vector is auto-parallel along the curve.

Let the principal bundle of bases $B\left(M^{n}\right)$ of a differentiable manifold $M^{n}$ be reduced to a totally disconnected group $K$. If $M^{n}$ has zero holonomy, that is $H\left(M^{n}, \Gamma\right)=0$, then $M^{n}$ is parallelizable by bases which are parallel under $\Gamma$.

Using parallel displacement we can establish a Riemannian metric in 
$M^{n}$. However the Riemann-Christoffel connection may not be $\Gamma$ and, indeed, the metric may not be flat unless the reduction of $B\left(M^{n}\right)$ to $K$ is realized.

Definition. Let $B\left(M^{n}\right)$ be reduced to a totally disconnected group $K$. Assume that on the holonomy covering space $\hat{M}^{n}$ the 1 -forms $\omega^{i}$, lying above the defining forms $\theta_{\alpha}^{i}$ on $M^{n}$, satisfy $d \omega^{i}=c_{j k}^{i} \omega^{i} \wedge \omega^{k}$ for real constants $c_{j k}^{i}, i, j, k$ $=1,2, \cdots, n$. Then the reduction of $B\left(M^{n}\right)$ to $K$ is called integrable.

Of course, each realizable reduction of $B\left(M^{n}\right)$ to $K$ is integrable for in this case the forms $\omega^{i}$ on $M^{n}$ satisfy $d \omega^{i}=0$.

In terms of the forms $\omega^{i}$ on $\hat{M}^{n}$, the natural Riemannian metric on $\hat{M}^{n}$ for a given reduction of $B\left(M^{n}\right)$ to a totally disconnected group $K$, is $d s^{2}$ $=\sum_{i=1}^{n}\left(\omega^{i}\right)^{2}$.

Theorem 8. Let $B\left(M^{n}\right)$ have an integrable reduction to a totally disconnected group $K$ and suppose that the corresponding Riemann metric on $\hat{M}^{n}$ is complete. Then the universal covering space $\tilde{M}^{n}$ is a Lie group and $\hat{M}^{n}$ is a corresponding homogeneous space. The Maurer-Cartan forms on $\tilde{M}^{n}$ are $\varpi^{i}$ which lie on $\tilde{M}^{n}$ above the $\omega^{i}$ of $\hat{M}^{n}$.

Proof. Now $\tilde{M}^{n}$ with the forms $\varpi^{i}$, which satisfy $d \varpi^{i}=c_{j k}^{i} \varpi^{j} \wedge \varpi^{k}$ has a complete Riemannian metric $d s^{2}=\sum_{i=1}^{i}\left(\varpi^{i}\right)^{2}$. Since $\tilde{M}^{n}$ is simply-connected, it is a Lie group [9] with left invariant forms $\varpi^{i}$ and structure constants $c_{j k}^{i}$.

Let $D$ be the covering transformation group of homeomorphisms of $\bar{M}^{n}$ over $\hat{M}^{n}$. Then $D$ preserves the forms $\varpi^{i}$ and thus consists of left translations [18] of $\tilde{M}^{n}$. We can consider $D \subset \tilde{M}^{n}$. Since $\tilde{M}^{n}$ is a covering space of $\hat{M}^{n}$, $D$ is discrete and furthermore the homogeneous space of right cosets $\widetilde{M}^{n} / D$ is differentiably isometric with $\hat{M}^{n}$. Q.E.D.

Corollary. The lie group $\tilde{M}^{n}$ is commutative if and only if the reduction of $B\left(M^{n}\right)$ to $K$ is realizable.

Proof. If $\tilde{M}^{n}$ is commutative, then $c_{j k}^{i}=0$ and $d \omega^{i}=0$ on $\hat{M}^{n}$. Then the projections of the forms $\omega^{i}$ in each local coordinate system of $M^{n}$, yield coordinates which realize the reduction of $B\left(M^{n}\right)$. The converse result was obtained in [5]. Q.E.D.

\section{BiBLIOGRAPHY}

1. W. Ambrose and I. Singer, A theorem on holonomy, Trans. Amer. Math. Soc. vol. 75 (1953) pp. 428-443.

2. L. Auslander, On holonomy covering spaces, Proc. Amer. Math. Soc. vol. 7 (1956) pp. 685-689.

3. - Examples of locally affine spaces, Ann. of Math. (1956) pp. 255-259.

4. L. Auslander and M. Kuranishi, Holonomy groups of locally euclidean spaces, Ann. of Math. (1957) pp. 411-415.

5. L. Auslander and L. Markus, Holonomy of flat affinely connected manifolds, Ann. of Math. vol. 62 (1955) pp. 139-152.

6. L. Auslander and I. Singer, Homogeneous locally affine spaces, (in preparation).

7. L. Bieberbach, Uber die Bewegungsgruppen der Euklidischen Raüme I, Math. Ann. voi. 70 (1911) pp. 297-336. 
8. - Uber die Bewegungsgruppen der Euklidischen Railme II, Math. Ann. vol. 72 (1912) pp. 400-412.

9. E. Cartan, La topologie des espaces representatifs des groupes de Lie, L'Enseignement Math. vol. 35 (1936) pp. 177-200.

10. S. S. Chern, Pseudo-groupes continus infinis, Colloques de Géométrie Differentielles, Strasbourg, 1953, pp. 119-136.

11. C. Ehresmann, Structures locales, Ann. Mat. Pura Appl. vol. 36 (1954) pp. 133-142.

12. L. Eisenhardt, Riemannian geometry, Princeton, 1949.

13. G. Hardy and E. Wright, An introduction to the theory of numbers, Oxford, 1938.

14. N. Kuiper, Sur les surfaces localement affines, Colloque de Géométrie Differentielle, Strasbourg, 1953, pp. 79-87.

15. L. Markus, Line element fields and Lorentz structures on differentiable manifolds, Ann. of Math. vol. 62 (1955) pp. 411-417.

16. J. Milnor, On the existence of a connection with curvature zero, (in preparation).

17. N. Steenrod, The topology of fiber bundles, Princeton, 1951. 771-776.

18. H. C. Wang, Complex parallisable manifolds, Proc. Amer. Math. Soc. vol. 5 (1954) pp.

Institute For Advanced StUdy,

Princeton, N. J.

Princeton University,

Princeton, N. J 